\title{
Considerations on the Design of a Molecular Frequency Standard Based on the Molecular Beam Electric Resonance Method*
}

\author{
Vernon W. Hughes \\ Gibbs Research Laboratories, Yale University, New Haven, Connecticut \\ (Received February 27, 1959; and in final form, May 30, 1959)
}

\begin{abstract}
The use of a rotational state transition as observed by the molecular beam electric resonance method is discussed as a possible frequency standard particularly in the millimeter wavelength range. As a promising example the $100 \mathrm{kMc}$ transition between the $J=0$ and $J=1$ rotational states of $\mathrm{Li}^{6} \mathrm{~F}^{19}$ is considered. The relative insensitivity of the transition frequency to external electric and magnetic fields and the low microwave power requirements appear favorable; the small fraction of the molecular beam that is in a single rotational state is a limiting factor.
\end{abstract}

\section{INTRODUCTION}

$I^{\mathrm{I}}$ recent years frequency standards based on microwave transitions in atoms and molecules have had great success. ${ }^{1}$ In particular, the cesium frequency standard ${ }^{2}$ which is based on the observation of a transition between the hyperfine structure levels of the cesium atom occurring at about $9192 \mathrm{Mc}$ by the atomic beam magnetic resonance method has been found to perform as an absolute frequency standard to an accuracy of about 1 part in $10^{10}$ over a long period of time. The ammonia maser ${ }^{3}$ which utilizes an inversion frequency at $23870 \mathrm{Mc}$ is less reliable as an absolute frequency standard because of the dependence of the output frequency on the resonant frequency of a cavity, but its relative short time stability has been observed to be as high as 1 part in $10^{12}$. The gas cell frequency standard, which is based on a magnetic dipole hyperfine structure transition in an alkali atom and which utilizes a buffer gas to reduce the line width, exhibits ${ }^{4}$ a large dependence of the transition frequency on the type and pressure of the buffer gas and hence does not appear easily capable of the absolute accuracy achieved by the cesium atomic beam frequency standard.

For the future development of more accurate frequency standards it is generally believed that the use of an atomic or molecular transition which occurs in the millimeter wavelength range may be important. The largest known ${ }^{5}$ hfs transition for a stable atom occurs for thallium, $\mathrm{Tl}^{205}$, at a frequency of $21311 \mathrm{Mc}$. A fine structure transition (for example, the ${ }^{2} P_{\frac{1}{2}} \rightarrow{ }^{2} P_{\frac{3}{2}}$ transition for the ground

* This work has been supported in part by the U. S. Army Signal Corps and the National Aeronautics and Space Administration.

1 Proceedings of Annual Symposia on Frequency Control for 1956, 1957, and 1958 of the U. S. Army Signal Corps Engineering Laboratories, Fort Monmouth, New Jersey.

${ }^{2}$ L. Essen and J. V. L. Parry, Trans. Roy. Soc. (London) 250, 45 (1957). The National Company of Malden, Massachusetts, now manufactures a cesium atomic frequency standard with a guaranteed absolute accuracy of about 5 parts in $10^{10}$.

${ }^{3}$ Gordon, Zeiger, and Townes, Phys. Rev. 99, 1264 (1955); Cedarholm, Bland, Havens, and Townes, Phys. Rev. Letters 1, 342 (1958).

${ }^{4}$ M. Arditi and T. R. Carver, Phys. Rev. 112, 449 (1958)

${ }^{5} \mathrm{P}$. Kusch and V. W. Hughes, "Atomic and molecular beam spectroscopy," Handbuch der Physik (Springer-Verlag, Germany, 1959), Vol. 37, p. 1. state of boron which occurs at a wavelength of $0.63 \mathrm{~mm}-$ may be found useful; however, the high power requirements for this transition and the inefficiency for detection of the atom are not encouraging.

The use of a rotational state transition in a molecule which can be observed by the molecular beam electric resonance method appears to offer some attractive possibilities, and it is the purpose of this article to discuss the design of a molecular frequency standard based on the molecular beam electric resonance method.

\section{MOLECULAR BEAM ELECTRIC RESONANCE METHOD}

The molecular beam electric resonance method ${ }^{5,6}$ is the electric analog of the well-known atomic or molecular beam magnetic resonance method which is used in the cesium atomic beam frequency standard. A schematic diagram of the molecular beam electric resonance method is shown in Fig. 1. Deflection of the molecule occurs through the electric force of an inhomogeneous electric field on the electric dipole moment of the molecule,

$$
F=\mu_{e} \nabla E .
$$

The effective electric dipole moment, $\mu_{e}$, depends on the rotational state of the molecule. If a transition is induced

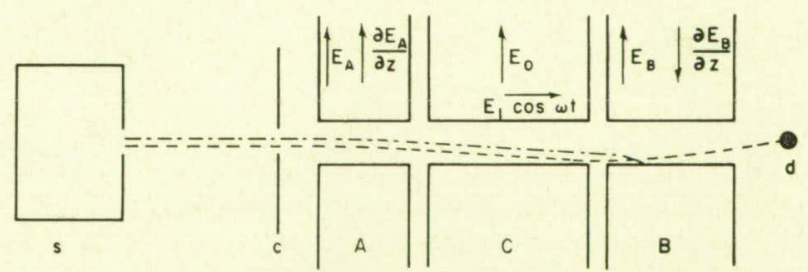

FIG. 1. Schematic diagram of a molecular beam electric resonance apparatus. The source chamber with source slit is designated by $s$. The collimator slit is designated by c. The A and $\mathrm{B}$ regions have inhomogeneous electric fields. The $\mathrm{C}$ region contains an oscillating electric field, $E_{1} \cos \omega t$, and in addition a constant and uniform electric field, $E_{0}$. The detector is designated by d. The dashed curve (---) is the trajectory of a molecule which remains in a single quantum state throughout its trajectory. The dot-dash curve $(-\cdot \cdot)$ is the trajectory of a molecule which undergoes a transition in quantum state in the $\mathrm{C}$ region.

${ }^{6}$ N. F. Ramsey, Molecular Beams (Clarendon Press, Oxford England, 1956). 
in the $\mathrm{C}$ region from one rotational state to another, it is observed through the change in trajectory of the molecule as a change in beam intensity at the detector. Thus far the electric resonance method has been applied only to alkali halide (and thallium halide) molecules, largely because these are the only molecules which are known to be detected with high efficiency by the hot wire surface ionization detector.

Design of the deflection system for an electric resonance apparatus requires a knowledge of the effective electric dipole moment, $\mu_{e}$, appearing in Eq. (1). This quantity is the electric dipole moment induced by the applied electric field and it depends on the rotational state, $J$, of the molecule and on the component, $m_{J}$, of rotational angular momentum in the field direction. The ratio of $\mu_{e}$ to the permanent electric dipole moment, $\mu$, is shown in Fig. 2 as a function of electric field for the rotational states $J=0,1$, and 2. Known values of the permanent electric dipole moments, $\mu$, of the alkali halide molecules are given in Table I. Thus far transitions have been studied only for rotational states $J=0,1$, and 2. Although there is no reason in principle why higher rotational states cannot be studied, in practice such experiments will be more difficult because $\mu_{e}$ is smaller for higher $J$ values. The magnitude of $\mu_{e}$ and of useful values of electric field are such that the deflection properties of a molecular beam electric resonance apparatus are similar to those of an atomic beam magnetic resonance apparatus where the atom has a magnetic moment of the order of a Bohr magneton.

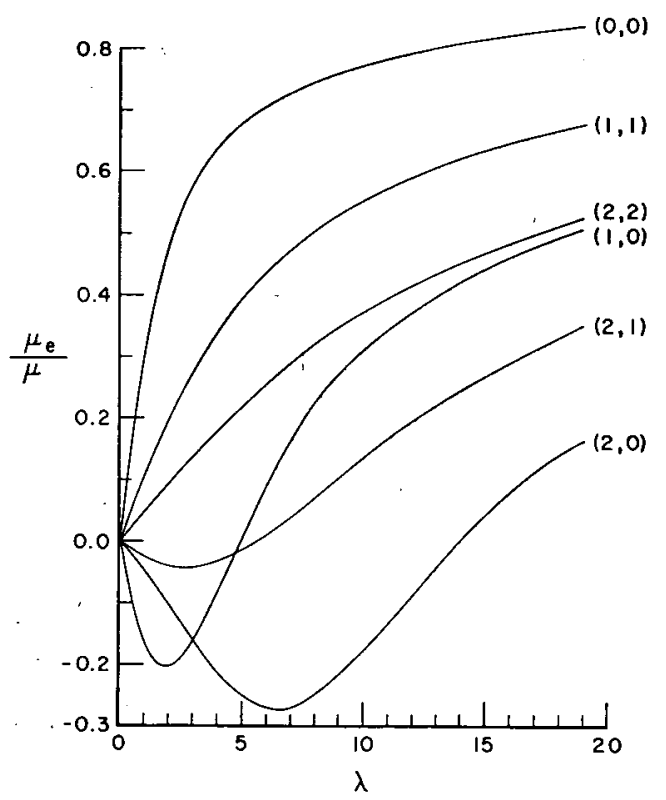

FIG. 2. Effective electric dipole moment, $\mu_{e}$, in units of the permanent electric dipole moment, $\mu$, vs the dimensionless parameter $\lambda\left(\lambda=\left(\mu E /\left(\hbar^{2} / 2 A\right)\right)\right.$, in which $E$ is the external electric field and $A$ is the moment of inertia of the molecule) for the rotational states $J=0, J=1$, and $J=2$. The different curves are designated by their $\left(J, m_{J}\right)$ values.
In the $\mathrm{C}$ region of a molecular beam electric resonance apparatus a static electric field is applied to maintain the state of quantization of the molecules. A microwave field is also applied to induce the electric dipole transition from one rotational state to another.

Designs of several molecular beam electric resonance apparatuses have been reported in detail in the literature. ${ }^{7-9}$

\section{MICROWAVE TRANSITIONS FOR ALKALI HALIDE MOLECULES}

Rotational state transitions have been studied for most of the alkali halide molecules either by the molecular beam electric resonance method, ${ }^{8}$ by conventional microwave absorption spectroscopy with a high-temperature spectroscope ${ }^{10}$ or by microwave absorption at millimeter wavelengths by a beam of alkali halide molecules. ${ }^{11}$ The frequency for a transition between the rotational states $J$ and $J+1$ is given approximately by the equation,

$$
\nu=2 B_{e}(J+1) \text {. }
$$

Table II gives values of the rotational constant $B_{e}$ for alkali halide molecules. ${ }^{5}$ In the cases designated by stars

TABLE I. Permanent electric dipole moments of alkali halide molecules (in debye or $10^{-18}$ esu units). ${ }^{\mathrm{a}}$

\begin{tabular}{lcccc}
\hline & $\mathrm{F}$ & $\mathrm{Cl}$ & $\mathrm{Br}$ & $\mathrm{I}$ \\
\hline $\mathrm{Li}$ & 6.6 & 5.9 & 6.2 & 6.6 \\
$\mathrm{Na}$ & & 8.5 & & 11.1 \\
$\mathrm{~K}$ & 7.3 & 10.5 & 10.4 & 12.1 \\
$\mathrm{Rb}$ & 8.8 & & & 10.5 \\
$\mathrm{Cs}$ & 7.9 & 10 & & 12 \\
\hline
\end{tabular}

a See reference 5 .

the $B_{e}$ values were determined from measurements of the spectrum at radio-frequencies, ${ }^{12}$ and no microwave rotational state transition has been observed.

Transitions at frequencies of $100 \mathrm{kMc}$ or higher are available as transitions between high $J$ rotational states for all the alkali halide molecules. Of especial interest is the $\mathrm{LiF}$ molecule for which the transition $J=0 \rightarrow J=1$ occurs at about $100 \mathrm{kMc}$.

A transition with a narrow line width can be obtained in a molecular beam apparatus provided the transit time of the molecule through the transition region is sufficiently long to give a small natural line width and provided line broadening due to inhomogeneity of external fields is small. In order to obtain a line width for an alkali halide molecule of about $200 \mathrm{cps}$ which is comparable to that of the cesium

${ }^{7}$ H. K. Hughes, Phys. Rev. 72, 614 (1947)

${ }^{8}$ Lee, Fabricand, and Rabi, Phys. Rev. 91, 1395 (1953).

${ }^{9}$ R. Braunstein and J. W. Trischka, Phys. Rev. 98, 1092 (1955).

${ }^{10}$ Honig, Mandel, Stitch, and Townes, Phys. Rev. 96, 629 (1954);

C. H. Townes and A. L. Schawlow, Microwave Spectroscopy (McGraw-

Hill Book Company, Inc., New York, 1955).

${ }^{11}$ A. K. Garrison and W. Gordy, Phys. Rev. 108, 899 (1957)

${ }^{12}$ J. C. Swartz and J. W. Trischka, Phys. Rev. 88, 1085 (1952). 
atomic beam frequency standard it is necessary to have a transition, region several hundred centimeters in length. Since this length is very long compared to the wavelength of the radiation, it would appear necessary to use the method of separated oscillating fields ${ }^{13}$ to induce the transition. This method employs two short regions with oscillating fields, one at the beginning and the other at the end of the transition region. It has the favorable property that the resonance frequency depends only on an average value of the external field in the $\mathrm{C}$ region (electric or magnetic) so that the line is not broadened by field inhomogeneities in the region between the two oscillating fields. The separated oscillating fields method has been successfully applied in many magnetic resonance experiments; it has not yet been used in electric resonance experiments, but no fundamental difficulties in its application are anticipated.

\section{USE OF $J=0 \rightarrow J=1$ TRANSITION IN $\mathrm{Li}^{6} \mathrm{~F}^{19}$ FOR A FREQUENCY STANDARD}

One of the most promising transitions for a frequency standard to operate at about $100 \mathrm{kMc}$ and based on the molecular beam electric resonance method is the transition $J=0 \rightarrow J=1$ for the $\mathrm{Li}^{6} \mathrm{~F}^{19}$ molecule. ${ }^{14}$ Detailed consideration of the energy levels, resonance line shape, and special features of the molecular beam electric resonance apparatus will be given for this case and should serve to illustrate more generally the design problems of a frequency standard based on the molecular beam electric resonance method.

The complete Hamiltonian for $\mathrm{LiF}$ which pertains to a rotational state transition can be written ${ }^{5,15}$

$$
\begin{aligned}
H=H^{(0)}(v, J)+c_{2} \mathbf{I}_{2} \cdot \mathbf{J}+c_{1} \mathbf{I}_{1} \cdot \mathbf{J} \\
-e q_{1} Q_{1} \frac{\left[3\left(\mathbf{I}_{1} \cdot \mathbf{J}\right)^{2}+\frac{3}{2}\left(\mathbf{I}_{1} \cdot \mathbf{J}\right)-\mathbf{I}_{1}{ }^{2} \mathbf{J}^{2}\right]}{2 I_{1}\left(2 I_{1}-1\right)(2 J-1)(2 J+3)} \\
+\frac{2 g_{1} g_{2} \mu_{0}^{2}}{R^{3}} \frac{\left[3\left(\mathbf{I}_{1} \cdot \mathbf{J}\right)\left(\mathbf{I}_{2} \cdot \mathbf{J}\right)-\left(\mathbf{I}_{1} \cdot \mathbf{I}_{2}\right) J(J+1)\right]}{(2 J-1)(2 J+3)} \\
-\mathbf{u} \cdot \mathbf{E}+\mu_{0} g_{1} \mathbf{I}_{1} \cdot \mathbf{H}+\mu_{0} g_{2} \mathbf{I}_{2} \cdot \mathbf{H}+\mu_{0} g_{J} \mathbf{J} \cdot \mathbf{H} .
\end{aligned}
$$

The term $H^{0}(v, J)$ gives the energy level of the molecule in the ground electronic state, in the vibrational state, $v$, and in the rotational state, $J$, in the absence of interactions which involve the nuclear spins or external fields. The interactions involving $c_{1}$ and $c_{2}$ (1 refers to the $\mathrm{Li}$ nucleus and 2 refers to the $F$ nucleus) are the spin-rotation interactions. ( $I_{1}$ and $I_{2}$ are the nuclear spins.) The term involving $e q_{1} Q_{1}$ is the electric quadrupole interaction for the $\mathrm{Li}$ nucleus. The term involving $g_{1} g_{2} \mu_{0}^{2} / R^{3}$ is the magnetic dipole-dipole interaction between the two nuclei

${ }^{13}$ N. F. Ramsey, Phys. Rev. 78, 695 (1950).

14 The natural abundance of the isotope $\mathrm{Li}^{6}$ is $7 \%$.

\begin{tabular}{|c|c|c|c|c|c|c|}
\hline & $F^{10}$ & $\mathrm{G}^{35^{\circ}}$ & $\mathrm{Cl}^{37}$ & $\mathrm{Br}^{79}$ & $\mathrm{Br}^{81}$ & $\mathrm{I}^{127} \mathrm{*}$ \\
\hline $\mathrm{Li}^{6}$ & $49000^{*}$ & $16000^{*}$ & $16000^{*}$ & & 19162.32 & 15381.99 \\
\hline $\mathrm{Li}^{7}$ & $44000^{*}$ & & & 16651.19 & 16617.62 & 13286.79 \\
\hline $\mathrm{Na}^{23}$ & & 6537.07 & & 4534.52 & 4509.35 & 3531.78 \\
\hline $\mathrm{K}^{39}$ & $6060^{*}$ & 3856.40 & 3746.61 & 2434.95 & 2415.08 & 1825.01 \\
\hline $\mathrm{K}^{41}$ & & 3767.42 & & & & \\
\hline $\mathrm{Rb}^{85}$ & 6315.64 & 2627.41 & & 1424.84 & & 984.32 \\
\hline $\mathrm{Rb}^{87}$ & 6289.10 & & & & & \\
\hline $\mathrm{Cs}^{133}$ & 5527.34 & 2161.21 & & 1081.34 & & 708.36 \\
\hline
\end{tabular}

${ }_{15}$ V. W. Hughes and L. Grabner, Phys. Rev. 79, 829 (1950).
TABLE II. Rotational constant, $B_{e}$, for alkali halide molecules (in Mc).

( $\mu_{0}$ is the Bohr magneton, the $g$ 's are the nuclear $g$ values expressed in the unit for which the electron spin $g$ value $\simeq+2$, and $R$ is the internuclear distance.) The term $-\boldsymbol{u} \cdot \mathbf{E}$ is the interaction of the electric dipole moment of the molecule with the static external electric field $\mathbf{E}$. The terms involving the static external magnetic field $\mathbf{H}$ are the interactions of the nuclear magnetic dipole moments and of the molecular rotational magnetic moment (characterized by $g_{J}$ ) with $\mathbf{H}$. No interaction of the form $\mathbf{I}_{1} \cdot \mathbf{I}_{2}$ is included since no such interaction has yet been observed for $\mathrm{LiF}$, although a small interaction of this type may occur. ${ }^{6}$

For the molecule $\mathrm{Li}^{6} \mathrm{~F}^{19}$ most of the constants appearing in Eq. (3) have been determined from the observation of radio-frequency transitions within the rotational states $J=1$ and $J=2$. The values of the constants together with their experimental uncertainties are given in the following: $c_{2} / h=(36.3 \pm 1.0) \times 10^{3} \mathrm{cps} ; \quad c_{1} / h=(0.8 \pm 0.5) \times 10^{3} \mathrm{cps}$,

$$
\begin{aligned}
\left|e q_{1} Q_{1}\right| / h & \leq(18.1 \pm 0.3) \times 10^{3} \mathrm{cps}, \\
g_{1} g_{2} \mu_{0}{ }^{2} /\left(h R^{3}\right) & =(4.8 \pm 2.0) \times 10^{3} \mathrm{cps}, \\
\mu & =(6.6 \pm 0.3) \times 10^{-18} \mathrm{esu}, \\
g_{1} & =-0.448 \times 10^{-3} ; \quad I_{1}=1, \\
g_{2} & =-2.863 \times 10^{-3} ; \quad I_{2}=\frac{1}{2}, \\
\left|g_{J}\right| & =(4.46 \pm 0.03) \times 10^{-5} .
\end{aligned}
$$

All the molecular constants refer to the $v=0$ vibrational state. The constants $c_{2}$ and $g_{J}$ were directly measured for $\mathrm{Li}^{6} \mathrm{~F}^{19}$ in molecular beam electric resonance experiments. ${ }^{2,16,17}$ The nuclear $g$ values, $g_{1}$ and $g_{2}$, were determined by nuclear magnetic resonance experiments. ${ }^{18}$ The constants $c_{1}$, eq $Q_{1} Q_{1}$, and $g_{1} g_{2} \mu_{0}^{2} /\left(h R^{3}\right)$ have not been directly measured. The values given were obtained from measurements of these constants for $\mathrm{Li}^{7} \mathrm{~F}^{19}$ by the molecular beam electric resonance method ${ }^{9}$ together with a knowledge of $g\left(\mathrm{Li}^{6}\right) / g\left(\mathrm{Li}^{7}\right)$ and of ${ }^{19} Q\left(\mathrm{Li}^{6}\right) / Q\left(\mathrm{Li}^{7}\right)$. The value of $\mu$ given in the foregoing is the value measured ${ }^{9}$ for $\mathrm{Li}^{7} \mathrm{~F}^{19}$.

\footnotetext{
${ }^{16}$ Kastner, Russell, and Trischka, J. Chem. Phys. 23, 1730 (1955)

17 A. M. Russell, Phys. Rev. 106, 1100 (1957).

${ }^{18}$ N. F. Ramsey, Nuclear Moments (John Wiley \& Sons, Inc., New York, 1953).

${ }_{19}$ P. Kusch, Phys. Rev. 75, 887 (1949)
} 
The rotational constant $B_{e}$ is known only through the determination $^{9}$ of the internuclear distance, $R$, from the measurement of the nuclear magnetic dipole-dipole interaction constant $g_{1} g_{2} \mu_{0}^{2} /\left(h R^{3}\right)$ for $\mathrm{Li}^{7} \mathrm{~F}^{19}$. $B_{\varepsilon}$ has the value (49 000 \pm 4000$)$ Mc.

The energy levels of the $\mathrm{Li}^{6} \mathrm{~F}^{19}$ molecule in zero electric and magnetic fields can be calculated by a perturbation theory based on the known relative orders of magnitude of the interaction terms,

$$
\left(\hbar^{2} / 2 A\right) \gg c_{2} \gg c_{1}, e q_{1} Q_{1}, \quad \text { or } \quad\left(g_{1} g_{2} \mu_{0}^{2} / R^{3}\right),
$$

in which $A$ is the moment of inertia of the molecule. The zero field representation defined by the quantum numbers $\left(I_{1}, I_{2}, J, F_{2}, F, M\right)$ is used in which $\mathbf{F}_{2}=\mathbf{I}_{2}+\mathbf{J}, \mathbf{F}=\mathbf{F}_{2}+\mathbf{I}_{\mathbf{1}}$, and $M$ is the component of $\mathbf{F}$ in the $z$ direction. The energy levels are given by the expression ${ }^{5,15}$

$$
\begin{aligned}
W=H_{0}(v, J)+\frac{c_{2} K_{2}}{2}+\frac{c_{1} K_{2}{ }^{\prime} K}{4 F_{2}\left(F_{2}+1\right)}+\frac{g_{1} g_{2} \mu_{0}{ }^{2}}{2 R^{3}(2 J-1)(2 J+3)}\left\{\frac{3 K_{2} K_{2}{ }^{\prime}}{2 F_{2}\left(F_{2}+1\right)}-\frac{J(J+1) K_{2}{ }^{\prime \prime}}{F_{2}\left(F_{2}+1\right)}\right\} K \\
\frac{-e q_{1} Q_{1}}{2 I_{1}\left(2 I_{1}-1\right)(2 J-1)(2 J+3)}\left\{3 M_{1}{ }^{2}+\frac{3}{8} \frac{K_{2}{ }^{\prime} K}{8 F_{2}\left(F_{2}+1\right)}-I_{1}\left(I_{1}+1\right) J(J+1)\right\},
\end{aligned}
$$

where

$$
\begin{aligned}
& K_{2}=F_{2}\left(F_{2}+1\right)-I_{2}\left(I_{2}+1\right)-J(J+1), \\
& K_{2}{ }^{\prime}=F_{2}\left(F_{2}+1\right)-I_{2}\left(I_{2}+1\right)+J(J+1) \text {, } \\
& K_{2}^{\prime \prime}=F_{2}\left(F_{2}+1\right)+I_{2}\left(I_{2}+1\right)-J(J+1), \\
& K=F(F+1)-I_{1}\left(I_{1}+1\right)-F_{2}\left(F_{2}+1\right), \\
& M_{1}{ }^{2}=\left\langle F_{2}, F, M\left|\left(I_{1} \cdot J\right)^{2}\right| F_{2}, F, M\right\rangle=M_{+}{ }^{2}+M_{0}{ }^{2}+M_{-}{ }^{2}, \\
& M_{+}{ }^{2}=\left[\frac{\left(F_{2}-I_{2}+J+1\right)\left(F_{2}+I_{2}-J+1\right)\left(F_{2}+I_{2}+J+2\right)\left(I_{2}+J-F_{2}\right)}{16\left(F_{2}+1\right)^{2}\left(2 F_{2}+1\right)\left(2 F_{2}+3\right)}\right] \\
& M_{0}{ }^{2}=\left[\frac{K_{2}^{\prime} K}{4 F_{2}\left(F_{2}+1\right)}\right]^{2} \\
& \times\left[\left(F+F_{2}-I_{1}+1\right)\left(F-F_{2}+I_{1}\right)\left(F+F_{2}+I_{1}+2\right)\left(F_{2}+I_{1}-F+1\right)\right], \\
& M_{-}{ }^{2}=\left[\frac{\left(F_{2}-I_{2}+J\right)\left(F_{2}+I_{2}-J\right)\left(F_{2}+I_{2}+J+1\right)\left(I_{2}+J-F_{2}+1\right)}{16 F_{2}{ }^{2}\left(2 F_{2}-1\right)\left(2 F_{2}+1\right)}\right] \\
& \times\left[\left(F+F_{2}-I_{1}\right)\left(F-F_{2}+I_{1}+1\right)\left(F+F_{2}+I_{1}+1\right)\left(F_{2}+I_{1}-F\right)\right] .
\end{aligned}
$$

The zero field energy levels for the $J=1$ state, obtained from Eq. (5) and the known interaction constants, are shown in Fig. 3. For the $J=0$ state there is only a single energy level. Each $F$ level has a $(2 F+1)$-fold degeneracy.

In the presence of an electric field the degeneracy of levels with different $|M|$ is removed. If the electric field is weak so that $\left(\mu^{2} E^{2} /\left(\hbar^{2} / 2 A\right)\right) \ll($ internal molecular interactions involving the $\mathrm{Li}^{6}$ nucleus) then the energy levels are obtained ${ }^{20}$ by the addition of a term of order $\mu^{2} E^{2} /\left(h^{2} / 2 A\right)$ to Eq. (5). For the $J=1$ levels with $F_{2}=\frac{1}{2}$ this quadratic Stark term actually has the coefficient zero. For the $J=0$ state the electric field dependence is given by

$$
W_{E}=-\mu^{2} E^{2} / 6\left(h^{2} / 2 A\right) .
$$

In the presence of a magnetic field the degeneracy is

${ }^{20}$ U. Fano, J. Research Natl. Bur. Standards, 40, 215 (1948). This paper gives the basic matrix elements required for calculation of the Stark effect (see also reference 15). completely removed. If the magnetic field is weak so that $\mu_{0} g_{2} H \ll$ (internal molecular interactions

involving the $\mathrm{Li}^{6}$ nucleus), then the energy contribution of the magnetic field is given by the addition to Eq. (5) of the term

$$
W_{H}=\mu_{0} g_{F} H M .
$$

The total $g$ value, $g_{F}$, is obtained from a generalized Landé formula for the three vector system of $\mathbf{I}_{1}, \mathbf{I}_{2}$, and $\mathbf{J}$ and will be of the order of a $g$ value for a nucleus $(\simeq-0.003)$.

A favorable transition for a frequency standard may be the transition from the $J=0$ state to the level designated by $\left(F_{2}=\frac{1}{2}, F=\frac{1}{2}\right)$ in the $J=1$ state, which will occur at a frequency of about $100 \mathrm{kMc}$. Both the upper and lower levels are single levels in the absence of a magnetic field. The upper level is sufficiently far from the adjoining levels in the $J=1$ state so that the resonance line shape of a 
200-cps wide line should be substantially unaffected by the presence of these other levels. The electric field dependence of the transition frequency is given from Eq. (6) as

$$
\nu=\nu_{0}+38 E^{2} \text { (cps), }
$$

in which $\nu_{0}$ is the transition frequency at zero field and $E$ is the field expressed in $\mathrm{v} / \mathrm{cm}$. Hence if the transition occurs in a field $E$ of $0.1 \mathrm{v} / \mathrm{cm}$, the frequency shift will be only 0.4 cps and a $10 \%$ change in the field will only alter the frequency by $0.1 \mathrm{cps}$. Transitions in such weak fields have been observed without undue loss of quantization. In order that the frequency shift due to a magnetic field $H$ shall be less than a few cycles per second, it is necessary to keep $H$ about 1 milligauss or less which should be possible.

. The specification of the line width will determine major features of the apparatus design. The vapor pressure of $\mathrm{LiF}$ is low and customarily an oven temperature of about $1200^{\circ} \mathrm{K}$ might be used. Hence the most probable velocity $\alpha$ for a molecule of mass $m$ in the oven will be given by

$$
\alpha=(2 k T / m)^{\frac{1}{2}}=0.9 \times 10^{5} \mathrm{~cm} / \mathrm{sec} .
$$

The line width of the central peak of the line obtained by separated, in-phase oscillating fields is given by

$$
\delta \nu=0.65 \alpha / L,
$$

in which $L$ is the distance between the two oscillating fields. Hence in order that $\delta \nu=200 \mathrm{cps}, L$ must be $300 \mathrm{~cm}$. The over-all length of the apparatus might then be some $400 \mathrm{~cm}$.

The microwave field required to induce the transition with near unity probability is determined from the condition, ${ }^{6}$

$$
V_{p q} \tau \simeq h .
$$

$V_{p q}$ is the amplitude of the matrix element of the interaction

$$
V=-\boldsymbol{u} \cdot \mathbf{E}_{1} e^{+i \omega t}
$$

of the microwave field $\mathbf{E}_{1} e^{+i \omega t}$ with the molecular electric dipole moment between the initial and final states, and $\tau$ is the time molecule $\alpha$ spends in an oscillating field region of length $l$. For a length $l$ of one $\mathrm{cm}$, the amplitude $E_{1}$ is of the order of $3 \times 10^{-4}$ esu or $0.1 \mathrm{v} / \mathrm{cm}$. Use of a cavity with dimensions of roughly $1 \mathrm{~cm}$ which operates in a higher mode and which has a $Q$ of 1000 would yield the proper amplitude of the microwave electric field provided the cavity is fed with a power of the order of a microwatt. Such an input power should be available from crystal harmonic generation from a klystron output. ${ }^{10}$

The principal difficulty in the use of the molecular beam electric resonance method as compared with the use of the magnetic resonance method is the reduced useful intensity

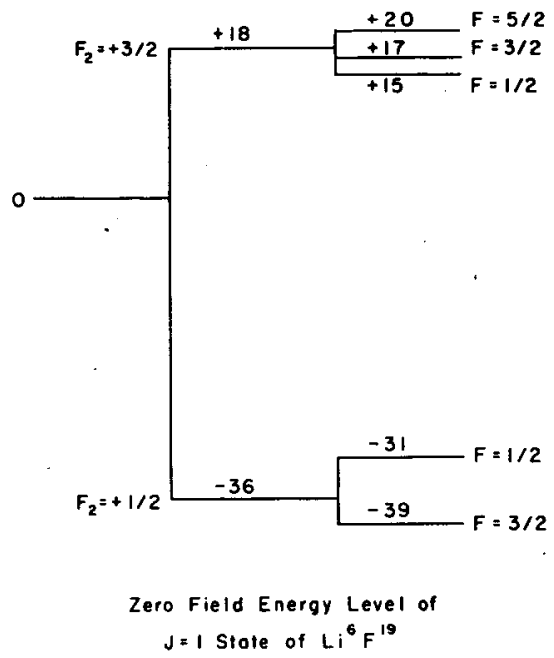

of the beam. The fraction of the molecules in the beam that are in the rotational state $J$ is given by ${ }^{21}$

$$
\left(N_{J} / N\right) \simeq(2 J+1)\left(h^{2} / 2 A k T\right),
$$

in which $T$ is the oven temperature and $k$ is Boltzmann's constant. For $\mathrm{Li}^{6} \mathrm{~F}^{19}$ with $T=1200^{\circ} \mathrm{K}, N_{0} / N=2 \times 10^{-3}$. It should be possible to utilize all the $J=0$ state molecules in the transition. For comparison, $\mathrm{Cs}$ has 16 hyperfine substates and only one is used for the transition in the National Company Atomichron. The useable oven pressure should be much the same as for cesium. The detection of $\mathrm{LiF}$ with high efficiency by the surface ionization process requires oxygenation of the hot wire unlike the detection of Cs.

In conclusion, the use of the transition $J=0 \rightarrow J=1$ in $\mathrm{Li}^{6} \mathrm{~F}^{19}$ as observed by the molecular beam electric resonance method appears favorable for obtaining a standard frequency in the $100-\mathrm{kMc}$ range, but the useful beam intensity will be less than that for a cesium atomic frequency standard due principally to the many rotational states present in the molecular beam.

The molecular beam electric resonance method may also prove useful in extending the frequency range of frequency standards by use of higher rotational state transitions than $J=0 \rightarrow J=1$. At present due to limitations of the efficiency of molecular detectors other than the surface ionization detector the molecular beam electric resonance method has been used only for alkali halide molecules. Further development of molecule detectors would extend the transitions available for a frequency standard using polar molecules and the molecular beam electric resonance method.

${ }^{21}$ G. Herzberg, Spectra of Diatomic Molecules (D. Van Nostrand Company, Inc., Princeton, New Jersey, 1950), p. 125. 